\title{
A PROBLEM ON THE RIESZ-DUNFORD OPERATOR CALCULUS AND CONVEX UNIVALENT FUNCTIONS
}

\author{
by J. S. HWANG
}

(Received 16 October, 1981)

Introduction. In his paper [3], Ky Fan asked whether if $f$ is a convex univalent function in the unit disk, with $f(0)=0$ and $f^{\prime}(0)=1$, then is it true that the set of $f(A)$ is a convex set of operators, when $A$ runs through all proper contractions on a Hilbert space? We answer this question in the negative.

Let $H$ be a complex Hilbert space. Let $A$ be an operator (i.e. a bounded linear transformation) on $H$ and let $\sigma(A)$ denote its spectrum. If $f(z)$ is a function analytic in a neighborhood $G$ of $\sigma(A)$, then $f(A)$ will denote the operator on $H$ defined by the usual Riesz-Dunford integral [2, p. 568]

$$
f(A)=\frac{1}{2 \pi i} \int_{C} f(z)(z I-A)^{-1} d z
$$

where $I$ stands for the identity operator on $H, C$ is a suitable finite family of positively oriented simple closed rectifiable contours.

As usual, an operator $A$ is called a contraction or a proper contraction, if its norm $\|A\| \leq 1$ or $\|A\|<1$ respectively.

Let $\Delta=\{z:|z|<1\}$ be the unit disk and let $K(\Delta)$ be the class of all convex univalent functions $f$ normalized by $f(0)=0$ and $f^{\prime}(0)=1$. Let $M(\Delta)$ be the class of all functions analytic in $\Delta$; then by a theorem of Brickman-MacGregor-Wilken [1], we know that the extreme points of the set $K(\Delta)$ in the vector space $M(\Delta)$ are precisely the functions of the form

$$
e_{\theta}(z)=z\left(1-e^{i \theta} z\right)^{-1}, \text { where } 0 \leq \theta<2 \pi .
$$

In [3, Theorem 8], Ky Fan proved that the set of all $e_{\theta}(A)$ is a convex set of operators, when $A$ runs through all proper contractions on a Hilbert space $H$. He then asked as to whether the same convexity is true for a function $f \in K(\Delta)$ instead of the extreme points. Furthermore, he proved that if $f$ is a starlike function then its operator range $f(A)$ is also starlike [3, Theorem 7]. From this, one might conjecture that the operator range $f(A)$ is convex if $f$ is convex. This however is false as will be seen from the following result. (For the definition of the Schwarz function, see [4, p. 385]).

THEOREM. The Schwarz function

$$
s(z)=\int_{0}^{z}\left(1-t^{4}\right)^{-1 / 2} d t=z+\sum_{1}^{\infty} \frac{1.3 \ldots(2 n-1)}{2^{n} n !(4 n+1)} z^{4 n+1}
$$

is convex and univalent in $\Delta$. Its operator range $s(A)$ is starlike but not convex.

Glasgow Math. J. 24 (1983) 129-130. 
Proof. The Schwarz function $s$ maps $\Delta$ conformally onto the square with vertices at $\pm s(1)$ and $\pm i s(1)$, so that it is convex and univalent in $\Delta$. To prove that the operator range $s(A)$ is not convex, we let

$$
A_{1}=r\left[\begin{array}{ll}
0 & 1 \\
1 & 0
\end{array}\right] \text { and } A_{2}=r\left[\begin{array}{rr}
0 & 1 \\
-1 & 0
\end{array}\right] \text {, where } 0<r<1
$$

Then the norm $\left\|A_{1}\right\|=\left\|A_{2}\right\|=r<1$. By a simple computation, we find that

$$
s\left(A_{1}\right)=\left[\begin{array}{cc}
0 & s(r) \\
s(r) & 0
\end{array}\right] \text { and } s\left(A_{2}\right)=\left[\begin{array}{cc}
0 & s(r) \\
-s(r) & 0
\end{array}\right] .
$$

If the assertion were false, then there would be a proper contraction $A$ such that

$$
s(A)=\frac{1}{2}\left(s\left(A_{1}\right)+s\left(A_{2}\right)\right)=\left[\begin{array}{cc}
0 & s(r) \\
0 & 0
\end{array}\right]=B .
$$

Since the function $w=s(z)$ is univalent and $s(0)=0$, it follows that the inverse $z=s^{-1}(w)$ is analytic at the origin and can be expanded as

$$
z=s^{-1}(w)=w+\sum_{2}^{\infty} a_{n} w^{n}
$$

This yields $A=s^{-1}(B)=B$, because $B^{n}=0$ for $n>1$.

Clearly, the function $s$ is continuous on the closure $\bar{\Delta}$ and the value $s(1)>1$. By choosing $r$ sufficiently close to 1 , we obtain the norm $\|A\|=\|B\|=s(r)>1$, a contradiction. This completes the proof.

\section{REFERENCES}

1. L. Brickman, T. H. MacGregor, and D. R. Wilken, Convex hulls of some classical families of univalent functions. Trans. Amer. Math. Soc. 156, (1971) 91-107.

2. N. Dunford and J. T. Schwartz, Linear operators, Part I: General theory. (Interscience, 1958).

3. Ky Fan, Analytic functions of a proper contraction, Math. Z. 160, (1978) 275-290.

4. E. Hille, Analytic function theory II. (Ginn, 1962).

INSTITUTE OF MATHEMATICS

ACADEmia Sinica

TAIPEI, TAIWAN. 